\title{
Archéopages
}

Archéopages

Archéologie et société

$35 \mid 10 / 2012$

Vivre avec les bêtes

\section{Relevé et restitution en 3D. Quel intérêt pour l'archéologie?}

\section{Simon Bryant}

\section{(2) OpenEdition}

1 Journals

Édition électronique

URL : https://journals.openedition.org/archeopages/315

DOI : 10.4000/archeopages.315

ISSN : 2269-9872

\section{Éditeur}

INRAP - Institut national de recherches archéologiques préventives

\section{Édition imprimée}

Date de publication : 1 mai 2013

Pagination : 84-87

ISSN : $1622-8545$

\section{Référence électronique}

Simon Bryant, «Relevé et restitution en 3D. Quel intérêt pour l'archéologie ? », Archéopages [En ligne], 35 | 10/2012, mis en ligne le 01 octobre 2014, consulté le 21 janvier 2022. URL : http:// journals.openedition.org/archeopages/315; DOI : https://doi.org/10.4000/archeopages.315 
l'ensemble 16 du site « Croc

au Loup n, à Étrechet, dans

I'Indre, permettant de tester

des hypothèses de travail

et reposant sur un protocole

particulier de fouille

(responsable d'opération

G. Robert, Inrap).

๘

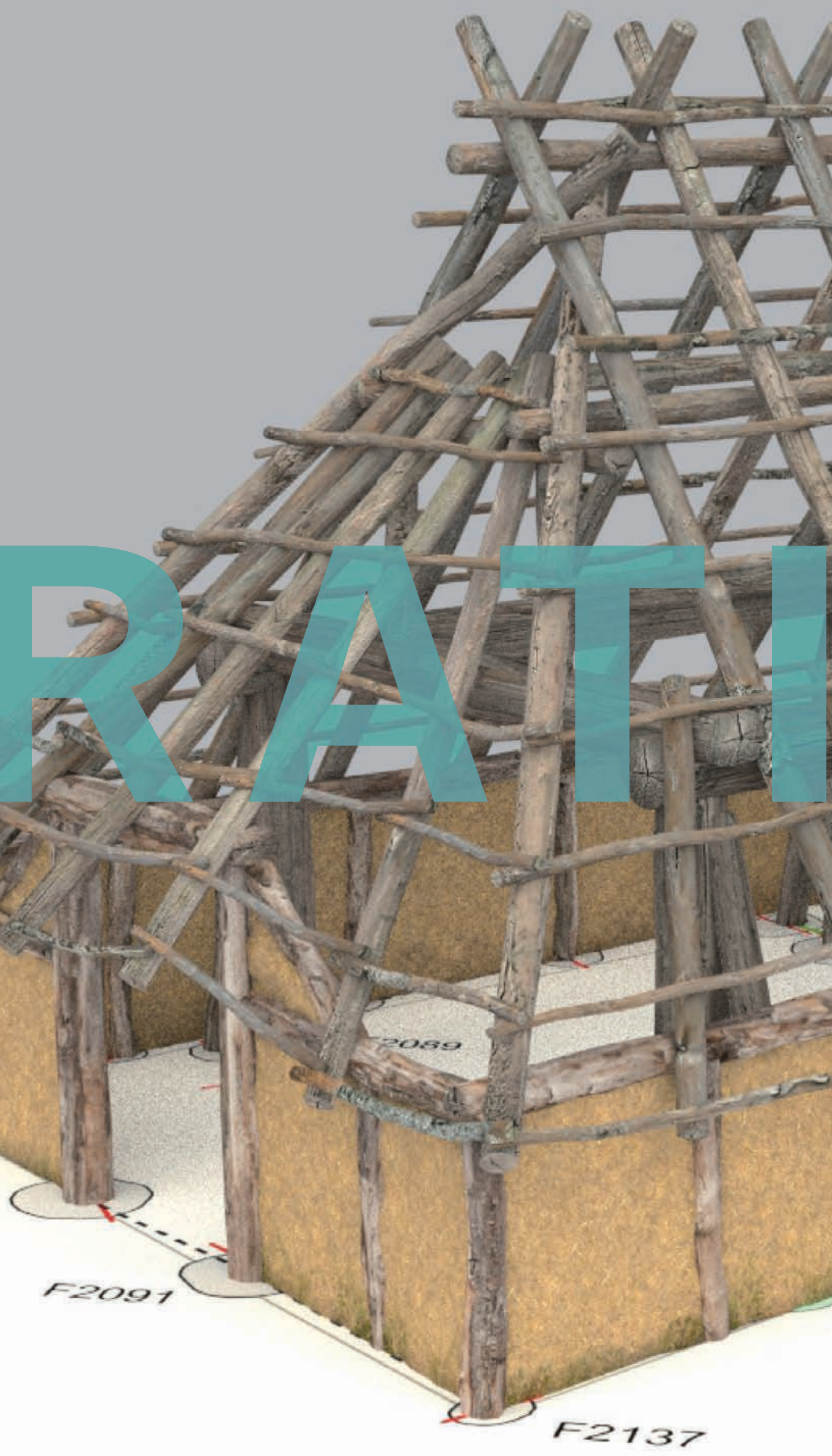


न4NIII
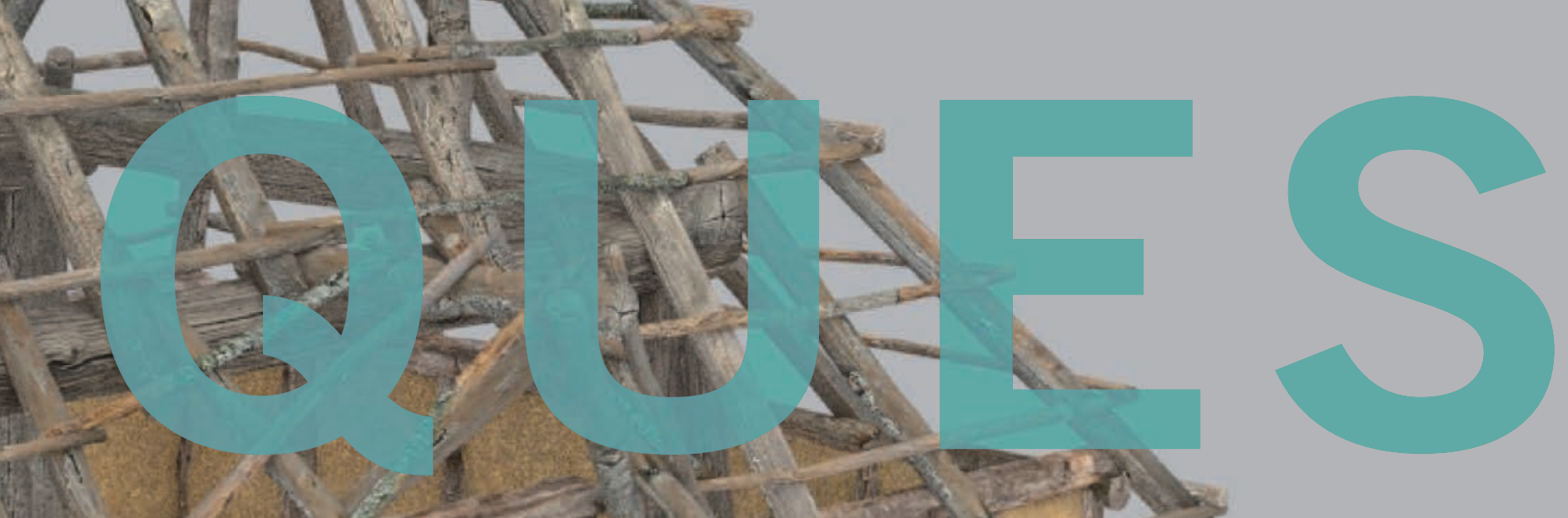

ชอ
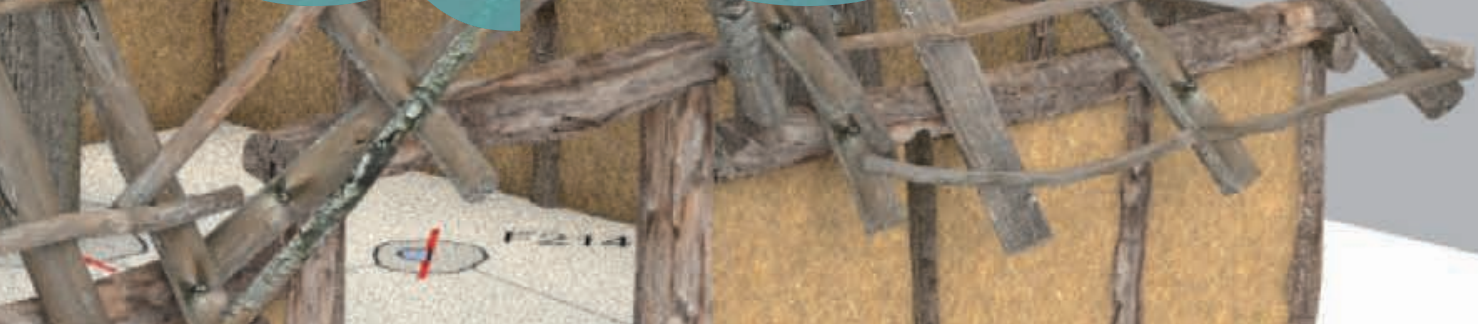

3.

,

$\frac{3}{4}$

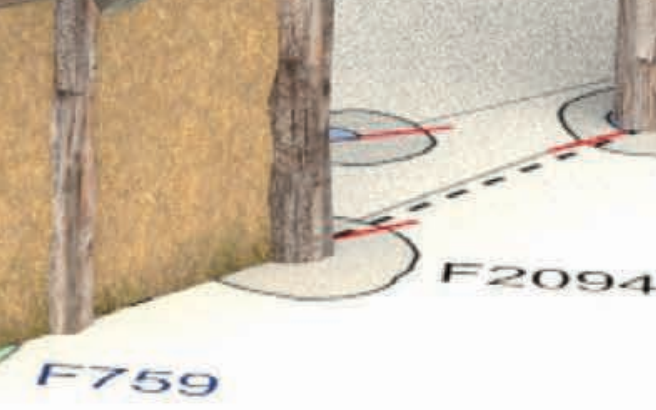




\section{L'image 3D comme aide à la démarche de restitution}

Après l'ensemble d'articles parus dans le numéro précédent sous le titre « Du bon usage de la $3 \mathrm{D}$ en archéologie », ceux-ci concernent plus particulièrement ce que cette technologie permet d'atteindre dans les projets de restitution. L'un des enjeux primordiaux de l'archéologie étant de rétablir rationnellement ce qui manque, l'image $3 \mathrm{D}$

\section{Relevé et restitution en $3 \mathrm{D}$. Quel intérêt pour l'archéologie? \\ Simon Bryant, Inrap}

Le tachéomètre et l'orthophotographie ont fait reculer la part du relevé manuel dans l'enregistrement archéologique, mais l'essentiel du relevé topographique et celui de la représentation des vestiges se fait «à plat» ou en deux dimensions, qu'il s'agisse des plans, des élévations ou des coupes. Pour des vestiges en élévation, ces documents peuvent servir à des restitutions de volumes et de niveaux, à l'aide de coupes ou de projections iso- ou axonométriques, ou grâce à la création de modèles en trois dimensions avec des logiciels de CAO tels AutoCad.

Une double méthode d'acquisition des points Les représentations obtenues par de telles méthodes sont souvent des simplifications du référent, dressées à partir des plans et des élévations. Il est également possible de restituer les objets en question d'après des nuages de points en trois dimensions acquis directement sur le terrain à l'aide des théodolites à rayon infrarouge ou laser.

Il faut distinguer deux approches pour l'acquisition des points. La première consiste à créer des volumes construits, que ce soit des maçonneries ou les espaces que celles-ci délimitent, telles les ouvertures.

1. Voir : http://cipa icomos.org/. 2. Une introduction sur les scans au laser peut être consultée sur : http://www.englishheritage.org.uk/ publications/3d-laserscanning-heritage2/. Voir aussi http://www.englishheritage.org.uk/ publications/measured and-drawn/ pour le relevé appliqué aux sites patrimoniaux.
Le relevé exige alors une grande rigueur dans la création des objets architecturaux qui doivent être «fermés», afin de constituer des volumes pleins ou vides, contrairement à un relevé en plan où une certaine tolérance existe à l'égard des traits isolés ou des objets « ouverts». Le nombre total de points relevés reste toutefois relativement faible. La seconde approche consiste à réaliser un scan en trois dimensions relevant une très grande quantité se révèle un support à la réflexion sur le rapport entre des vestiges, toujours singuliers, et un modèle, général, démarche qui permet tout autant de définir les vestiges que d'affiner le modèle et qui constitue l'étape préalable indispensable lorsqu'il s'agit de reconstruire concrètement des monuments.

de points par le balayage automatisé d'un tachéomètre au laser. Un logiciel de CAO traite les points obtenus afin de créer des surfaces et des objets qui peuvent être visualisés. Le niveau de détail varie en fonction du type d'objet et de la distance, mais les résolutions sont suffisamment élevées pour permettre la modélisation de petits objets tels des sculptures, jusqu'aux ensembles monumentaux de grande taille : ces modélisations numériques constituent alors les premières archives des édifices dans le cadre de leur préservation. Le risque demeure néanmoins de considérer le relevé comme un but en soi et la réalisation de modèles en trois dimensions comme un enregistrement total de l'édifice en question (Buehrer et al., 2001). Le CIPA est l'un des comités internationaux de l'ICOMOS (International Council on Monuments and Sites), établi en collaboration avec l'ISPRS (International Society of Photogrammetry and Remote Sensing). La table ronde du CIPA ${ }^{\mathbf{1}}$ (International Scientific Committee for Documentation of Cultural Heritage) à Potsdam en 2001 était centrée sur les méthodes de relevé et de cartographie du patrimoine, mais l'absence d'archéologues parmi les intervenants et le nontraitement de l'aspect archéologique illustrent bien le clivage entre patrimoine et archéologie. La photogrammétrie architecturale constituait l'orientation initiale dans un contexte de conservation du patrimoine, domaine où l'archéologie est assez peu représentée par les archéologues de terrain ${ }^{2}$ (Buehrer et al., 2001; Saint-Aubin, 2001 ; Böhler et al., 2004 ; Barber, Mills, 2007). L'application des méthodes de photogrammétrie à l'archéologie a été abordée par plusieurs congrès à partir de celui de Krakow (1990), dont Göteborg (1997), Potsdam (2001), Athènes (2009) et Kyoto (2009).

L'utilisation de relevés en trois dimensions dans le cadre des interventions archéologiques est moins 

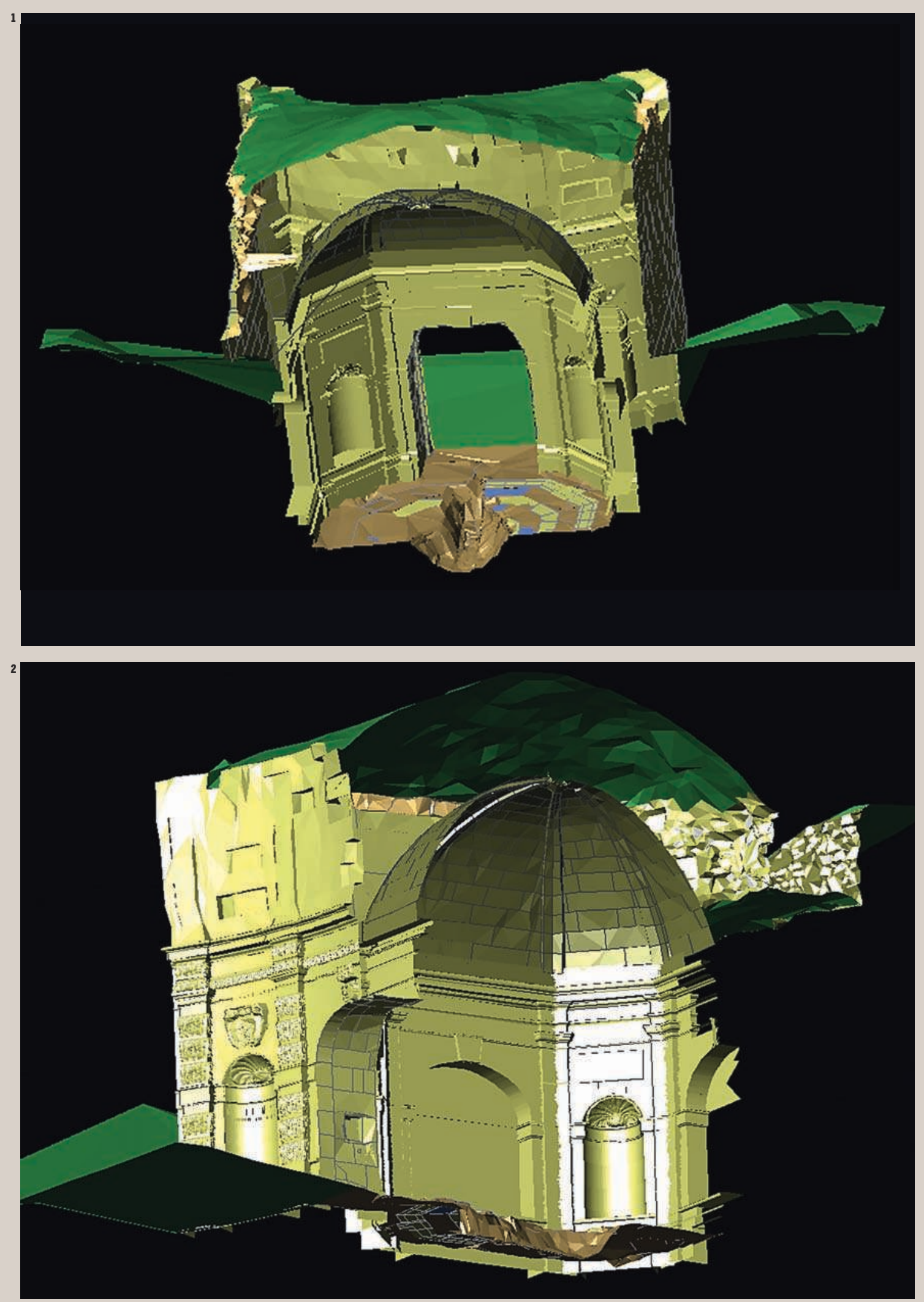

1. Le Grand-Pressigny (Indreet-Loire), nymphée du château.

Vue perspective restituée de

l'intérieur du pavillon depuis

l'arrière de l'édifice en

direction de l'esplanade,

devant la façade principale.

2.zVue en coupe avec volume

restitué de la moitté orientale

du pavillon, vue de dessous. 
fréquente. D'une part, les moyens techniques nécessaires sont encore assez onéreux et, d'autre part, l'étude archéologique d'un édifice en élévation se fonde avant tout sur l'analyse stratigraphique de l'ensemble avec un objectif et une méthodologie bien distincts de l'approche patrimoniale. Dans ce contexte, le relevé en plan et en élévation est généralement suffisant pour créer des supports graphiques à fins archéologiques. Toutefois, le relevé en trois dimensions possède quelques attraits que nous illustrerons à travers deux études d'édifices en élévation.

\section{Un outil du diagnostic archéologique} Le premier concerne l'évaluation du nymphée du château du Grand-Pressigny (Indre-et-Loire), une grotte architecturale abritant un système hydraulique du début du XVII ${ }^{\mathrm{e}}$ siècle. L'intervention réalisée en 2007 (Bryant, 2007) devait préciser la nature du site préalablement à tout projet de restauration et de mise en valeur, et consistait en une fouille partielle des vestiges du système hydraulique à l'intérieur du bâtiment et sur l'esplanade devant la façade principale. Plusieurs facteurs, dont la relative instabilité de la partie haute des murs, ont poussé à privilégier la réalisation d'orthophotographies pour les élévations, d'autant que les maçonneries représentent une seule campagne de construction tout en possédant un montage et un décor complexes. Le relevé des vestiges dégagés par la fouille a été fait entièrement avec le tachéomètre, en combinant des orthophotographies et des représentations en correspondance avec les US enregistrées. Le choix d'abandonner le relevé manuel découlait à la fois des possibilités offertes par les moyens techniques et d'un désir de garder une certaine cohérence dans l'enregistrement: il s'agissait d'éviter les relevés sur support papier en vue de leur intégration a posteriori dans le fonds de plan topographique. Les vestiges de l'intérieur ont été relevés au théodolite et les données ont été affinées en utilisant les orthophotographies de l'emprise de la fouille à l'intérieur comme à l'extérieur. En revanche, les difficultés à assurer un éclairage régulier de l'intérieur ont limité l'utilisation de l'orthophotographie comme document de travail. La création d'un modèle en trois dimensions [ill. 1-2] représente la suite logique de cet enregistrement numérique. Elle était fondée sur un relevé «hybride», alliant la représentation des volumes principaux des maçonneries et des composants architectoniques avec celle de certains éléments de décor répétitifs à partir des nuages de points (procédé similaire à celui des scans automatisés). L'utilité du modèle ne résidait pas tant dans la visualisation des niveaux des sols, des adductions et des plans d'eau, mais plutôt dans la mise en évidence des relations entre les principales composantes du site et de la topographie immédiate à partir d'angles de vue différents. Le fonds de plan peut également servir de base pour une restitution d'éléments disparus (grand bassin devant la façade principale ou hypothétique mur-écran fermant l'esplanade). Le modèle numérique a été réalisé sur la base d'un nombre de points bien inférieur aux nuages de points générés par des scans automatiques et le rendu des surfaces a été fait sous AutoCad: la qualité graphique du rendu est donc bien loin des images de synthèse produites par des logiciels dédiés aux graphismes en $3 \mathrm{D}$. De ce point de vue, il s'agit d'une expérience destinée à tester une approche rarement utilisée par les archéologues dans le cadre préventif et avec des moyens techniques et humains réduits. Dans le cas de la poursuite éventuelle des fouilles, il est à espérer que la modélisation en trois dimensions pourra être complétée, notamment pour l'environnement et la topographie générale du parc, paysage construit selon les contraintes du relief et de l'hydrologie locale. La reprise du travail sera facilitée par l'archivage numérique des vestiges enregistrés.

\section{Un outil pour la compréhension du bâti} Le second exemple de l'utilisation d'un relevé en trois dimensions porte sur la tour et les terrasses de Foix et les latrines de l'aile Louis XII du château de Blois (Loir-et-Cher). Ce projet de recherches programmées a commencé en 2009 ; l'étude de la tour et des terrasses de Foix a eu lieu en 2010 et celle des latrines de Louis XII en 2011 (Bryant et al., 2010 et 2012). Dans les deux cas, l'objectif consistait à fournir une documentation archéologique sur l'état général de conservation d'un ensemble dont la topographie médiévale avait été fortement transformée par des aménagements et des constructions du XVII ${ }^{\mathrm{e}}$ siècle. Le choix du relevé en trois dimensions avait été dicté par le terrain, dont les contraintes rendaient quasi impossible la projection des axes de dessin sur les niveaux des bâtiments ainsi que le positionnement de leurs parties : le temps nécessaire au relevé manuel aurait compromis l'analyse archéologique et les marges d'erreur auraient été trop importantes pour fournir un fonds de plan exploitable ultérieurement. La modélisation en trois dimensions a rendu plus évidentes les relations entre les divers volumes et les aménagements des différentes époques, notamment pour la tour de Foix, dont le premier étage d'origine avaient été transformé en rez-dechaussée $\mathrm{au} \mathrm{XV}^{\mathrm{e}}$ siècle, puis condamné et en partie englobé par les constructions du XVII ${ }^{\mathrm{e}}$ siècle. Tout comme pour le site du nymphée, il manque, pour terminer l'étude $3 \mathrm{D}$, des données topographiques et archéologiques complémentaires. La qualité des images demeure encore décevante, mais la création de ces objets virtuels sert des finalités scientifiques et non pas esthétiques. De fait, bien que les relevés «à plat» s'avèrent généralement suffisants, la modélisation en trois dimensions présente d'autres attraits : elle constitue le point de départ d'un archivage numérique évolutif qui peut servir de base aux données archéologiques quelles qu'elles soient, comme nos relevés habituels. Dans le secteur du patrimoine bâti, elle pourrait devenir 
un puissant outil de compréhension des ensembles bâtis complexes ou disparus, grâce aux possibilités offertes : la visualisation à partir de différents points de vue, l'extraction de coupes ou de vues écorchées, et la possibilité d'ajouter ou de masquer des structures appartenant à différentes phases de construction. La 3D permet également une présentation pédagogique au public de vestiges disparus ou inaccessibles, notamment par les bornes interactives dans les programmes muséographiques ou sur les sites patrimoniaux accessibles via internet.

Le sous-investissement des archéologues dans la $3 \mathrm{D}$ tient peut-être au fait que les relevés sont réalisés par des cabinets spécialisés. Dès lors, les archéologues risquent de se voir marginalisés face à la tendance du «tout technique » de ces cabinets d'ingénieurs, capables de fournir des belles images mais sans réelle valeur archéologique ou historique car créées sans analyse technique et stratigraphique. Or ces démarches constituent justement la particularité de l'archéologie par rapport aux autres métiers du patrimoine. Les clients de ces services de $3 \mathrm{D}$, essentiellement des collectivités, sont-ils suffisamment informés pour connaître les limites des méthodes et du produit fourni? Lappropriation de ces techniques par les archéologues permettra d'assurer la validité des restitutions et de constituer une archive numérique évolutive. Les chantiers préventifs ont d'ores et déjà démontré les apports de l'archéologie dans le domaine du patrimoine bâti.

\section{Références bibliographiques}

BARBER D., MiLLS J., 2007, 3 D laser scanning for heritage. Advice and guidance to users on laser scanning in archaeology and architecture, English Heritage, Londres.

BöHLER W., HeInz G., Marbs A., 2004, « The Potential of Non-Contact Close Range Laser Scanners for Heritage Recording Purposes », in Geoinformatics 2004, Proceedings of the $12^{\text {th }}$ International Conference on Geoinformatics. Geospatial Information Research: Bridging the Pacific and Atlantic, Actes du colloque de l'Universitéde Gävle (Suède), 7-9 Juin 2004, p. 291-298.

BRYANT S., 2007, Le Grand-Pressigny, 37.113.034.AH Indre-et-Loire, Région Centre. Le nymphée du château. Une architecture et un système hydraulique de la Renaissance, Rapport de diagnostic archéologique, Inrap, Orléans.

Bryant S., Aubourg V. (collab.), Philippe M. (collab.), WEDAJO B. (COLLAB.), 2010, Blois (41 - Loir-et-Cher), le Château. Programme d'opération archéologique pluriannuelle, campagne 2010. Campagne de relevés archéologiques des élévations de la tour de Foix et du bastion dit de Marie de Médicis, rapport archéologique, Inrap, Orléans.

Bryant S., Boukef Y. (collab.), Prevot A. (collab.), WEDAJO B. (COLLAB.), 2012, Blois (41 - Loir-et-Cher), le Château. Les latrines de Louis XII, première approche archéologique des élévations, rapport d'étude archéologique, Inrap, Orléans.

Buehrer Th., Fraser C., Gruen A., Li Z., Ruther H., 2001, « Photogrammetric reconstruction and ${ }_{3} \mathrm{D}$ visualisation of Bet Giorgis, a rock-hewn church in Ethiopia ", in Proceedings of the XVIII CIPA Symposium, Postdam, 18 -21 Septembre 2001, article en ligne, http://cipa.icomos.org/text\%2ofiles/ potsdam/2001-07-tbo2.pdf, consulté le 14 mars 2001

Coordination: Sylvie Deffressigne, Inrap. 2. Inrap, architecte de formation.

3. Étudiant à l'école nationale supérieure d'Architecture de Nancy.
La restitution $3 \mathrm{D}$ comme outil de recherche archéologique.

L'exemple du bâtiment antique de Bouxières-sous-Froidmont

Karine Boulanger, Inrap, EA 1132

« Histoire et cultures de l'Antiquité et du Moyen Âge »

En archéologie, la restitution 3D n'est pas exclusivement réservée à la conception d'illustrations pédagogiques esthétiques destinées à la valorisation des sites auprès du grand public. Elle peut également être conçue comme un outil essentiel à l'approfondissement de la réflexion des chercheurs. L'expérience menée à Bouxières-sous-Froidmont (Meurthe-et-Moselle), au lieu-dit «Le Tremble», témoigne de l'intérêt de cette démarche.

Cette fouille archéologique préventive, réalisée en 2003 , a livré un établissement rural isolé dont l'occupation s'échelonne entre le milieu du i ${ }^{\mathrm{er}}$ et le milieu du $\mathrm{III}^{\mathrm{e}}$ siècle. Ce bâtiment sur fondations en pierre bénéficiait de niveaux de sol bien conservés. Létude des vestiges a notamment permis de caractériser les différents espaces et de préciser la nature des matériaux et des techniques constructives des élévations [cf. article p. 34-41]. Dans l'espace interne de la grange, pièce principale de la construction, la présence de trous de poteaux a particulièrement retenu l'attention des fouilleurs [ill. 1]. Ces indices d'une structure porteuse, contemporaine des murs maçonnés, ont ouvert le débat sur les différentes possibilités de restitution des élévations. Afin de pousser la réflexion à son terme et démontrer la compatibilité d'un bâtiment sur fondations en pierre avec une structure porteuse sur poteaux de bois, nous avons tenté l'exercice d'une restitution 3D. Cette expérience a été rendue possible grâce à la collaboration de René Elter ${ }^{2}$ et à la participation bénévole de Sylvain Poilprez ${ }^{3}$.

Dans un premier temps, le travail de restitution a été guidé par la mise en évidence des schémas régulateurs régissant le plan de l'édifice. La grange et ses aménagements internes sont tracés à partir d'un module carré de base, de 10 pieds de côté [ill. 1, tracé vert]. Le développement des espaces périphériques postérieurs est quant à lui basé sur le report d'un module rectangulaire correspondant à la moitié de la superficie de la construction initiale [ill. 1, tracé rouge]. Dans un second temps, il est apparu que la restitution d'élévations en pan de bois sur soubassement maçonné était la plus appropriée à notre cas de figure [ill. 2]. En effet, les poteaux internes semblent destinés à soutenir le plancher d'un étage conçu comme une structure indépendante, partiellement liée aux élévations de la grange [ill. 3]. Cette méthode constructive suggère que les murs périphériques sont réalisés en structure légère. L'existence de parois en torchis est par ailleurs suggérée par la découverte de couches d'argile crue et de torchis brûlé dans les niveaux d'abandon du bâtiment. Le choix de la hauteur des élévations 